\title{
Left-sided Breast Cancer Irradiation With Deep Inspiration Breath-hold: Changes in Heart and Lung Dose in Two Periods
}

\author{
ANNE CAROLINE KNÖCHELMANN, NESE CEYLAN and MICHAEL BREMER
}

Clinic for Radiation Therapy and Special Oncology, Hannover Medical School, Hannover, Germany

\begin{abstract}
Background/Aim: Post-operative radiotherapy for breast cancer can increase cardiac disease in a dosedependent manner. In this study we show the reduction of dose to heart and left anterior descending artery (LAD) which can be achieved by using "Deep inspiration breathhold" (DIBH) technique. Patients and Methods: Tangential $3 D$-planned radiation was delivered to 357 patients with leftsided breast cancer, 159 of them with the DIBH technique. A distinction was made according to fractionation scheme. Results: The mean heart dose was significantly reduced by DIBH from $2.64 \mathrm{~Gy}$ to $1.39 \mathrm{~Gy}(p<0.001)$. The mean dose to the LAD was significantly reduced from $5.68 \mathrm{~Gy}$ to $3.88 \mathrm{~Gy}$ $(p<0.001)$. Mean dose and volume receiving 5, 10 and $15 \mathrm{~Gy}$ of ipsilateral lung were higher with both hypofractionated schedule and conventional fractionation in the DIBH group. Conclusion: DIBH in left-sided breast irradiation is an effective method of reducing the radiogenic heart dose.
\end{abstract}

One of the most common malignant tumours in women is breast cancer (1). After surgical therapy, postoperative radiotherapy reduces the risk of local recurrence and is therefore an obligatory part of breast conserving therapy. While long-term survival has been shown to be improved (2), long-term cardiac toxicity of different therapeutic approaches requires greater attention.

In left-sided breast cancer, adjuvant radiotherapy may be associated with an increased risk of cardiac injury (3). Conventional tangential treatment technique may influence potential cardiac risk when parts of the heart and in particular the left anterior descending artery (LAD) are

This article is freely accessible online.

Correspondence to: Anne Caroline Knöchelmann, Hannover Medical School, Clinic for Radiation Therapy and Special Oncology, Carl-Neuberg Str. 1, 30625 Hannover, Germany. Tel: +49 17615327341, email: knoechelmann.anne@mh-hannover.de

Key Words: Breast cancer, deep inspiration breath-hold (DIBH), 3D-planned tangential radiotherapy (3D-CRT). located within the treatment field. Any reduction in radiation exposure to the heart may lower the risk of ischemic heart disease. One simple and readily available method to reduce the dose to the heart is the deep inspiration breath-hold technique (4).

The objective of this analysis was to evaluate treatment planning and compare dosimetry plans in free breathing; non-deep inspiration breath-hold (nDIBH) and deep inspiration breath-hold (DIBH), with the aim to validate the findings of Darby in 2013 (3) within our own cohort of patients based on individual CT-based three-dimensional treatment planning. In order to define acceptable doses for the heart and LAD, it is necessary to evaluate potential dosimetric benefits of DIBH while maintaining acceptable lung toxicity.

This will be achieved, in this retrospective study, using comparison of quantitatively dose-volume exposure of the heart and LAD as well as the ipsilateral lung. This study included patients with left-sided breast cancer receiving postoperative radiotherapy with 3D-planned tangential fields at our Department using either DIBH or nDIBH. The key issue is whether the systematic application of DIBH could consistently reduce radiation exposure of the heart and LAD below the postulated dose limits by Darby (3) and whether this also applies to modern hypofractionated radiation schedules. Consequently, two different time periods, before and after the publication of Darby in 2013 (3) were compared, resulting in more awareness of avoiding radiation exposure to the heart and subsequent use of the DIBH technique in left-sided breast cancer patients.

\section{Patients and Methods}

Patient characteristics, radiation treatment plans and information on cardiac risk factors were collected retrospectively from databases of the department of radiation oncology of the Hannover Medical School. The conduct of the study was reviewed and approved by the local ethics committee.

In total, 357 ongoing patients with left-sided breast cancer were included in the study; of which 159 were irradiated using DIBH technique. Inclusion criteria for this study was 3D-planned tangential radiotherapy (3D-CRT) in case of curative breast conserving therapy 
Table I. Patient baseline characteristics of the study cohort $(n=357)$.

\begin{tabular}{|c|c|c|c|}
\hline & $\mathrm{nDIBH}$ & $\mathrm{DIBH}$ & Total \\
\hline Number of patients & 198 & 159 & 357 \\
\hline Median age (range) & $60.7(24-88)$ & $56.7(24-79)$ & 58.9 \\
\hline \multicolumn{4}{|l|}{ Histology } \\
\hline Ductal invasive & 145 & 112 & 257 \\
\hline Lobular invasive & 26 & 13 & 39 \\
\hline DCIS & 22 & 29 & 51 \\
\hline Others & 5 & 5 & 10 \\
\hline \multicolumn{4}{|l|}{ Tumour stage } \\
\hline pT1 & 96 & 69 & 165 \\
\hline pT2 & 53 & 30 & 83 \\
\hline pT3 & 14 & 7 & 21 \\
\hline pT4 & 11 & 0 & 11 \\
\hline pTis & 22 & 29 & 51 \\
\hline Unknown & 2 & 24 & 26 \\
\hline \multicolumn{4}{|l|}{ Hormonal receptor } \\
\hline Positive & 174 & 118 & 292 \\
\hline Negative & 24 & 41 & 65 \\
\hline \multicolumn{4}{|l|}{ Her2neu-receptor } \\
\hline Positive & 19 & 16 & 35 \\
\hline Negative & 179 & 143 & 322 \\
\hline \multicolumn{4}{|l|}{ Histopathological grading } \\
\hline Well & 32 & 26 & 58 \\
\hline Moderate & 106 & 70 & 176 \\
\hline Poor & 60 & 63 & 123 \\
\hline \multicolumn{4}{|l|}{ Surgery } \\
\hline Breast conserving & 168 & 134 & 302 \\
\hline Mastectomy & 30 & 25 & 55 \\
\hline $\begin{array}{l}\text { Potential cardiotoxic drugs } \\
\text { (Epirubicin, Cyclophosphamid, } \\
\text { Taxane) }\end{array}$ & 56 & 46 & 102 \\
\hline Herceptin & 19 & 16 & 35 \\
\hline Antihormonal therapy & 158 & 103 & 261 \\
\hline \multicolumn{4}{|l|}{ Radiotherapy } \\
\hline Conventional fractionation & 150 & 24 & 174 \\
\hline Hypofractionated schedule & 48 & 135 & 183 \\
\hline Sequential Boost to tumour bed & ed 75 & 69 & 144 \\
\hline Periclavicular lymph nodes & 45 & 31 & 76 \\
\hline
\end{tabular}

nDIBH: Non-deep inspiration breath-hold, DIBH: deep inspiration breath-hold, DCIS: ductal carcinoma in situ.

( $\mathrm{n}=302 ; 168$ in the $\mathrm{nDIBH}$ group and 134 in the DIBH group) or mastectomy ( $\mathrm{n}=55 ; 30$ in the $\mathrm{nDIBH}$ group; 25 in the DIBH group), respectively. The ipsilateral periclavicular lymph nodes without internal mammary nodes were irradiated in 76 patients (45 in the $\mathrm{nDIBH}$ group and 31 in the DIBH group). All patients were irradiated postoperatively in supine position. Patients receiving partial breast irradiation or re-irradiation were excluded from the study.

Patients with left-sided breast cancer were grouped according to their respective time period of radiation treatment. First group included a total of 198 patients irradiated without DIBH (nDIBH) between $01 / 2011$ and $06 / 2013$, second group included a total of 159 patients irradiated with DIBH between 01/2017 and 12/2019 after consequent clinical implementation of DIBH technique. Within the same period a transition took place from conventional fractionation (CF) to more routinely application of hypofractionated radiotherapy schedules (HF) according to changes of clinical practice guidelines. Baseline clinical and treatment related parameters of patients in both groups (nDIBH and DIBH) are listed in Table I.

All patients received a noncontrast enhanced free-breathing planning computed tomography (CT) scan in supine position with $3 \mathrm{~mm}$ axial slice thickness. Postoperative surgical scars were marked with radiopaque material. From 2017 onwards all patients with left-sided breast cancer with close positional relationship of the heart to the chest wall received a second planning CT scan with DIBH during the same appointment. Decision to use DIBH was based on a visually expected benefit compared to free breathing. Main criterion for choosing DIBH technique was whether the medial aspects of the anticipated tangential radiation field borders would cross the heart in free breathing planning CT.

A simple breath-hold technique (non-computer controlled) was used during radiotherapy. After initial patient coaching amplitude of breathhold was monitored by visual control via camera outside by medical staff (audio-visual guidance). For consistency and to reduce inter observer variability the heart, LAD and lungs were retrospectively delineated on planning CT by the same physician (Anne Caroline Knöchelmann). The heart as well as the LAD was delineated according to the CT-based atlas by Feng (5). The cranial border of the heart was contoured beginning underneath the pulmonary trunk, ascending aorta and superior vena cava. The lower border of the heart was the caudal myocardial border near the diaphragm. All fatty tissue within the pericardium was contoured because of the presence of cardiac vessels. The LAD was contoured from the left coronary artery underneath the right pulmonary vessel running between right and left ventricle to cardiac apex. Therefore, on some images the LAD was not visible in noncontrast enhanced CT scan. The location of the LAD was then inferred using visible landmarks. Contouring of the left lung was carried out excluding the bronchial tubes.

Clinical target volume (CTV) was delineated according to Radiation Therapy Oncology Group breast cancer atlas (6). The CTV included all ipsilateral breast tissue except muscles below and adjacent skin. In case of chest wall irradiation, the posterior border of the CTV ended at the anterior pleural surface including the ribs and intercostal muscles. The margin for the planning target volume (PTV) was 5-10 mm taking into account adjustments on the medial and lateral as well as inferior and superior borders. A sequential radiation boost was applied in patients younger than 50 years or presence of risk factors (e.g., poor grading). The boost volume was delineated on the basis of available pre-operative imaging supported by radiopaque marking of surgical scars.

All patients were treated with 3D-CRT by tangential fields using a linear accelerator with individual multileaf collimation. Tangential irradiation beams, with segmental fields for dose homogenization if necessary, were used for breast or chest wall treatment with $6 \mathrm{MV}$ photons and if necessary $15 \mathrm{MV}$ photons for partial fields in selected cases.

Quantitative dose-volume parameters of heart, LAD and ipsilateral lung were extracted from the treatment planning system (Oncentra ${ }^{\circledR}$ by Elekta, V4.3). To analyse differences between the dose-volume parameters in the $\mathrm{nDIBH}$ and DIBH group, statistical analyses were performed. The following parameters were derived from the dosevolume histogram of each treatment plan: absolute volume of contoured heart, the mean heart dose (MHD) as well as partial volume parameters of the heart receiving 5 Gy (V5), 10 Gy (V10) and 25 Gy (V25); For LAD we sampled the absolute volume of LAD as well as maximum dose $\left(\mathrm{LAD} \mathrm{D}_{\max }\right)$ and mean dose $\left(\mathrm{LAD} \mathrm{D}_{\text {mean }}\right)$; 
Table II. Volumes of organs at risk (volume in $\left.\mathrm{cm}^{3}\right)$.

\begin{tabular}{|c|c|c|c|c|c|c|}
\hline & \multicolumn{2}{|c|}{ All $(n=357)$} & \multicolumn{2}{|c|}{ nDIBH $(\mathrm{n}=198)$} & \multicolumn{2}{|c|}{ DIBH $(n=159)$} \\
\hline & Mean \pm SD & Range & Mean \pm SD & Range & Mean \pm SD & Range \\
\hline Heart & $502 \pm 135$ & 195-1099 & $561 \pm 129$ & 291-1099 & $429 \pm 103$ & $195-884$ \\
\hline LAD & $2.93 \pm 0.89$ & $0.76-6.02$ & $2.95 \pm 1.01$ & $0.76-6.02$ & $2.9 \pm 0.71$ & $1.57-5.02$ \\
\hline Ipsilat. Lung & $1614 \pm 573$ & $630-3435$ & $1223 \pm 323$ & $630-2262$ & $2102 \pm 424$ & $956-3435$ \\
\hline PTV & $986 \pm 471$ & $111-3196$ & $998 \pm 487$ & $268-3196$ & $971 \pm 451$ & $111-2215$ \\
\hline
\end{tabular}

nDIBH: Non-deep inspiration breath-hold; DIBH: deep inspiration breath-hold; LAD: Left anterior descending artery; Ipsilat.: ipsilateral; PTV: planning target volume; SD: standard deviation; n.s.: non-significant.

Table III. Dosimetric parameters of heart, LAD and ipsilateral lung.

\begin{tabular}{|c|c|c|c|c|c|c|}
\hline & \multicolumn{2}{|c|}{$\mathrm{nDIBH}$} & \multicolumn{2}{|c|}{ DIBH } & \multirow[b]{2}{*}{ Diff abs. (rel.) } & \multirow[b]{2}{*}{$p$-Value } \\
\hline & Mean \pm SD & Range & Mean \pm SD & Range & & \\
\hline \multicolumn{7}{|l|}{ Heart } \\
\hline MHD (Gy) & $2.64 \pm 1.26$ & $0.83-10.85$ & $1.39 \pm 0.47$ & $0.59-3.11$ & $-1.25(-47 \%)$ & $<0.001$ \\
\hline V5 (\%) & $6.63 \pm 5.61$ & $0.29-42.45$ & $2.09 \pm 1.98$ & $0.01-10.20$ & $-4.55(-69 \%)$ & $<0.001$ \\
\hline V10 (\%) & $3.21 \pm 3.03$ & $0.03-21.94$ & $1.26 \pm 1.21$ & $0.02-6.49$ & $-1.95(-61 \%)$ & $<0.001$ \\
\hline V25 (\%) & $2.20 \pm 3.63$ & $0.01-25.08$ & $0.67 \pm 0.80$ & $0.01-3.44$ & $-1.53(-69 \%)$ & $<0.001$ \\
\hline \multicolumn{7}{|l|}{ LAD } \\
\hline $\mathrm{D}_{\max }(\mathrm{Gy})$ & $12.43 \pm 12.18$ & $1.61-49.25$ & $11.05 \pm 9.18$ & $2.11-36.94$ & $-1.38(-11 \%)$ & n.s. \\
\hline $\mathrm{D}_{\text {mean }}(\mathrm{Gy})$ & $5.68 \pm 5.24$ & $1.57-49.13$ & $3.88 \pm 2.59$ & $1.36-18.24$ & $-1.80(-32 \%)$ & $<0.001$ \\
\hline \multicolumn{7}{|l|}{ Lung } \\
\hline MLD (Gy) & $6.49 \pm 2.83$ & $1.61-18.23$ & $5.92 \pm 2.23$ & $1.68-12.72$ & $-0.57(-9 \%)$ & n.s. \\
\hline V5 (\%) & $18.34 \pm 8.05$ & $1.00-44.24$ & $25.21 \pm 8.82$ & $7.19-51.43$ & $6.87(37 \%)$ & $<0.001$ \\
\hline V10 (\%) & $14.31 \pm 6.78$ & $2.33-38.81$ & $16.65 \pm 6.55$ & $3.24-33.46$ & $2.34(16 \%)$ & $<0.001$ \\
\hline V15 (\%) & $12.71 \pm 6.32$ & $1.04-36.84$ & $13.07 \pm 5.6$ & $1.80-28.08$ & $0.36(3 \%)$ & n.s. \\
\hline V20 (\%) & $11.49 \pm 5.91$ & $0.63-34.87$ & $10.91 \pm 5.11$ & $1.08-25.00$ & $-0.59(-5 \%)$ & n.s. \\
\hline
\end{tabular}

nDIBH: Non-deep inspiration breath-hold; DIBH: deep inspiration breath-hold; LAD: Left anterior descending artery; Gy: Gray; MHD: mean heart dose; MLD: mean lung dose; $\mathrm{V}(\mathrm{x})$ : volume receiving (x) Gy of the dose; SD: standard deviation; n.s.: non-significant.

for the lung the volume of the ipsilateral lung was sampled, mean dose of ipsilateral lung (MLD) and relative volume of the ipsilateral lung receiving 5 Gy (V5), 10 Gy (V10), 15 Gy (V15) and 20 Gy (V20). Details are displayed in Table II and Table III.

All data were tested for normal distribution using the Kolmogorov Smirnov test $(\alpha=0.05)$. Variables with normal distribution were analysed using unpaired $t$-test. Variables without normal distribution were analysed using Mann-Whitney $U$-test. Values of $p<0.05$ were considered to be statistically significant. Correlations between different parameters were determined using linear regression and quantified as Pearson's correlation coefficient. SPSS Statistics 28 was used for the statistical analysis.

\section{Results}

Main findings regarding comparative dose exposure of heart, LAD and left lung are described below. Detailed results are shown in Table II, Table III and Table IV. For the complete cohort of patients mean heart volume was $502 \pm 135 \mathrm{~cm}^{3}$ (range $=195-1,099 \mathrm{~cm}^{3}$ ) and mean volume of LAD was $2.93 \pm 0.89 \mathrm{~cm}^{3}$ (range $\left.=0.76-6.02 \mathrm{~cm}^{3}\right)$. Mean lung volume was $1,614 \pm 573 \mathrm{~cm}^{3}$ (range $=630-3,435 \mathrm{~cm}^{3}$ ) (Table II).

In the nDIBH group the MHD was $2.64 \pm 1.26 \mathrm{~Gy}$ and in the DIBH group the MHD was $1.39 \pm 0.47$ Gy (Table III). Taking into account the fractionation scheme, in patients with conventional fractionation (CF), MHD in the $\mathrm{nDIBH}$ group was $2.84 \pm 1.32$ Gy and in the DIBH group $1.81 \pm 0.52$ Gy. In patients with hypofractionated schedule (HF), the MHD in the nDIBH group was $2.03 \pm 0.81 \mathrm{~Gy}$ and in the DIBH group $1.31 \pm 0.42$ Gy (Table IV).

Using DIBH resulted in a significant reduction in MHD in both $\mathrm{CF}$ (reduction of $1.03 \mathrm{~Gy} ; p<0.001$ ) and $\mathrm{HF}$ (reduction of $0.72 \mathrm{~Gy} ; p<0.001$ ) using DIBH (Table IV; Figure 1). In the $\mathrm{nDIBH}$ group, 42 patients $(21 \%)$ received a MHD greater than 3.0 Gy and 9 patients $(5 \%)$ received 
Table IV. Dosimetric parameters of heart, LAD and ipsilateral lung depending on fractionation regimen (Conventional fractionation and hypofractionated schedule).

\begin{tabular}{|c|c|c|c|c|c|c|c|c|}
\hline & \multicolumn{4}{|c|}{ Conventional fractionation } & \multicolumn{4}{|c|}{ Hypofractionated schedule } \\
\hline & \multirow{2}{*}{$\begin{array}{c}\text { nDIBH } \\
\text { Mean } \pm \text { SD }\end{array}$} & \multirow{2}{*}{$\begin{array}{c}\text { DIBH } \\
\text { Mean } \pm \text { SD }\end{array}$} & \multicolumn{2}{|c|}{ nDIBH $v s$. DIBH } & \multirow{2}{*}{$\begin{array}{c}\text { nDIBH } \\
\text { Mean } \pm \text { SD }\end{array}$} & \multirow{2}{*}{$\begin{array}{c}\text { DIBH } \\
\text { Mean } \pm \text { SD }\end{array}$} & \multicolumn{2}{|c|}{ nDIBH $v s$. DIBH } \\
\hline & & & Diff abs. (rel.) & $p$-Value & & & Diff abs. (rel.) & $p$-Value \\
\hline \multicolumn{9}{|l|}{ Heart } \\
\hline MHD (Gy) & $2.84 \pm 1.32$ & $1.81 \pm 0.52$ & $-1.03(-36 \%)$ & $<0.001$ & $2.03 \pm 0.81$ & $1.31 \pm 0.42$ & $-0.72(-35 \%)$ & $<0.001$ \\
\hline $\mathrm{V} 5(\%)$ & $7.35 \pm 5.89$ & $3.12 \pm 2.54$ & $-4.24(-58 \%)$ & $<0.001$ & $4.33 \pm 3.81$ & $1.87 \pm 1.78$ & $-2.46(-57 \%)$ & $<0.001$ \\
\hline $\mathrm{V} 10(\%)$ & $3.30 \pm 3.15$ & $1.66 \pm 1.54$ & $-1.65(-50 \%)$ & $<0.001$ & $2.87 \pm 2.58$ & $1.14 \pm 1.09$ & $-1.73(-60 \%)$ & $<0.001$ \\
\hline $\mathrm{V} 25(\%)$ & $2.37 \pm 3.93$ & $1.01 \pm 0.86$ & $-1.36(-57 \%)$ & n.s. & $1.59 \pm 2.16$ & $0.53 \pm 0.75$ & $-1.05(-66 \%)$ & $<0.001$ \\
\hline \multicolumn{9}{|l|}{ LAD } \\
\hline $\mathrm{D}_{\max }(\mathrm{Gy})$ & $13.59 \pm 12.96$ & $13.43 \pm 10.31$ & $-0.16(-1 \%)$ & n.s. & $8.82 \pm 8.47$ & $10.63 \pm 8.94$ & $1.82(21 \%)$ & 0.038 \\
\hline $\mathrm{D}_{\text {mean }}(\mathrm{Gy})$ & $6.30 \pm 5.73$ & $4.41 \pm 2.04$ & $-1.89(-30 \%)$ & n.s. & $3.75 \pm 2.45$ & $3.79 \pm 2.67$ & $0.04(1 \%)$ & n.s. \\
\hline \multicolumn{9}{|l|}{ Lung } \\
\hline MLD (Gy) & $7.03 \pm 2.94$ & $8.53 \pm 2.24$ & $1.50(21 \%)$ & 0.007 & $4.80 \pm 1.50$ & $5.46 \pm 1.90$ & $0.66(14 \%)$ & 0.017 \\
\hline V5 (\%) & $19.61 \pm 8.51$ & $34.22 \pm 7.32$ & $14.6(74 \%)$ & $<0.001$ & $14.37 \pm 4.53$ & $23.61 \pm 8.08$ & $9.24(64 \%)$ & $<0.001$ \\
\hline V10 (\%) & $15.13 \pm 7.22$ & $23.40 \pm 5.84$ & $8.27(55 \%)$ & $<0.001$ & $11.73 \pm 4.27$ & $15.45 \pm 5.92$ & $3.71(32 \%)$ & $<0.001$ \\
\hline V15 (\%) & $13.42 \pm 6.76$ & $18.58 \pm 5.22$ & $5.16(38 \%)$ & $<0.001$ & $10.50 \pm 4.00$ & $12.09 \pm 5.09$ & $1.59(15 \%)$ & 0.030 \\
\hline V20 (\%) & $12.17 \pm 6.28$ & $16.19 \pm 4.84$ & $4.02(33 \%)$ & 0.001 & $9.40 \pm 3.91$ & $9.97 \pm 4.57$ & $0.57(6 \%)$ & n.s. \\
\hline
\end{tabular}

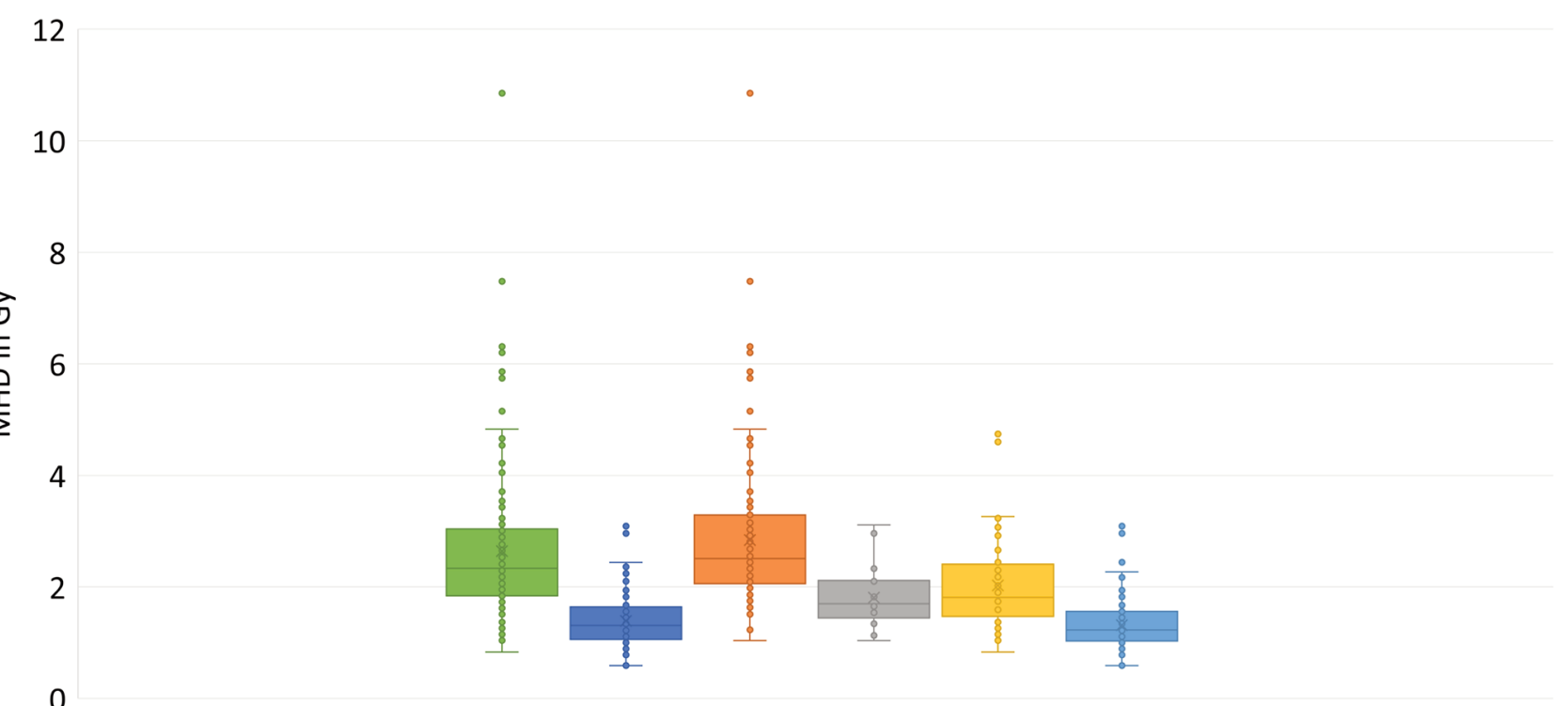

\section{$\square \mathrm{nDIBH}$ total $\square \mathrm{DIBH}$ total $\square \mathrm{nDIBH}$ CF $\square \mathrm{DIBH}$ CF $\square \mathrm{nDIBH} \mathrm{HF} \square \mathrm{DIBH} \mathrm{HF}$}

Figure 1. Mean heart dose (MHD) in DIBH compared to nDIBH. Gy: Gray; CF: conventional fractionation; HF: hypofractionated schedule; nDIBH: non-deep inspiration breath-hold; DIBH: deep inspiration breath-hold.

more than 5.0 Gy. By contrast, in the DIBH group only 2 patients $(1 \%)$ received a MHD greater than $3.0 \mathrm{~Gy}$. Both latter patients received postmastectomy irradiation, one of them with $\mathrm{CF}$ and the other with $\mathrm{HF}$. Besides the reduction of MHD, the volumes of the heart receiving $5 \mathrm{~Gy}$ and $10 \mathrm{~Gy}$ (V5 and V10) were significantly reduced by DIBH
( $p \leq 0.001$ ), while V25 was significantly reduced in patients with HF only (Table IV).

In the $\mathrm{nDIBH}$ group the LAD $\mathrm{D}_{\text {mean }}$ was $5.68 \pm 5.24 \mathrm{~Gy}$ and in the DIBH group it was $3.88 \pm 2.59$ Gy (Table III). In the $\mathrm{CF} / \mathrm{nDIBH}$ group, LAD $\mathrm{D}_{\text {mean }}$ was $6.30 \pm 5.73 \mathrm{~Gy}$ and in the $\mathrm{CF} / \mathrm{DIBH}$ group $4.41 \pm 2.04 \mathrm{~Gy}$. In the $\mathrm{HF} / \mathrm{nDIBH}$ group, 


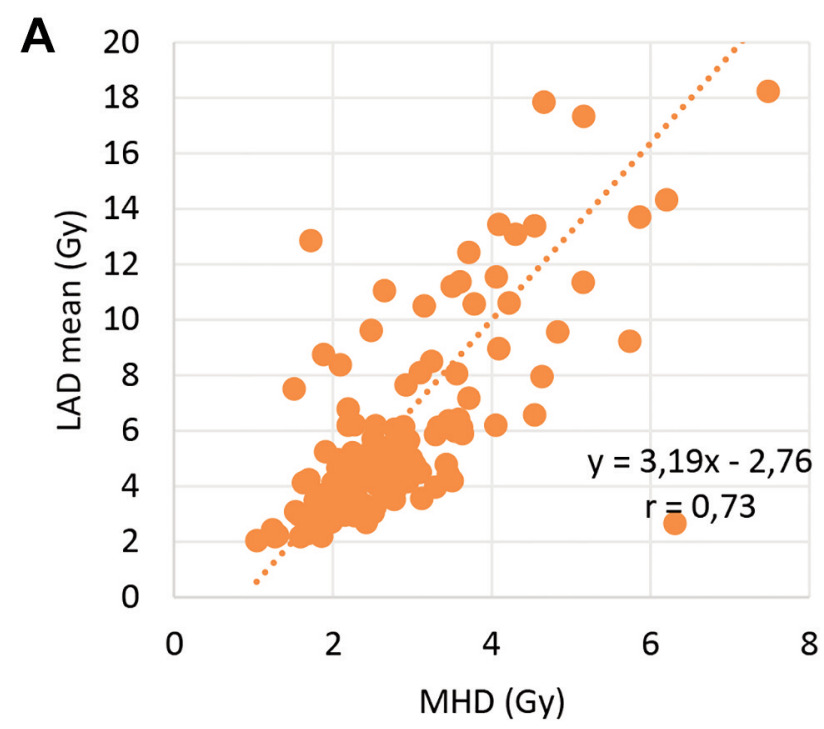

nDIBH CF $\ldots \ldots$ Linear (nDIBH CF)

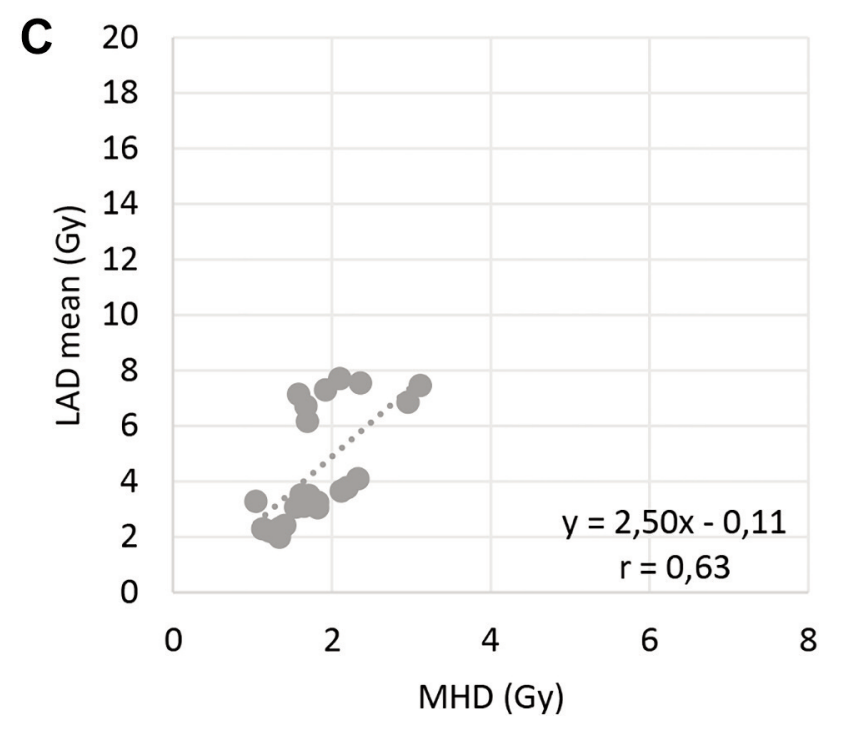

DIBH CF

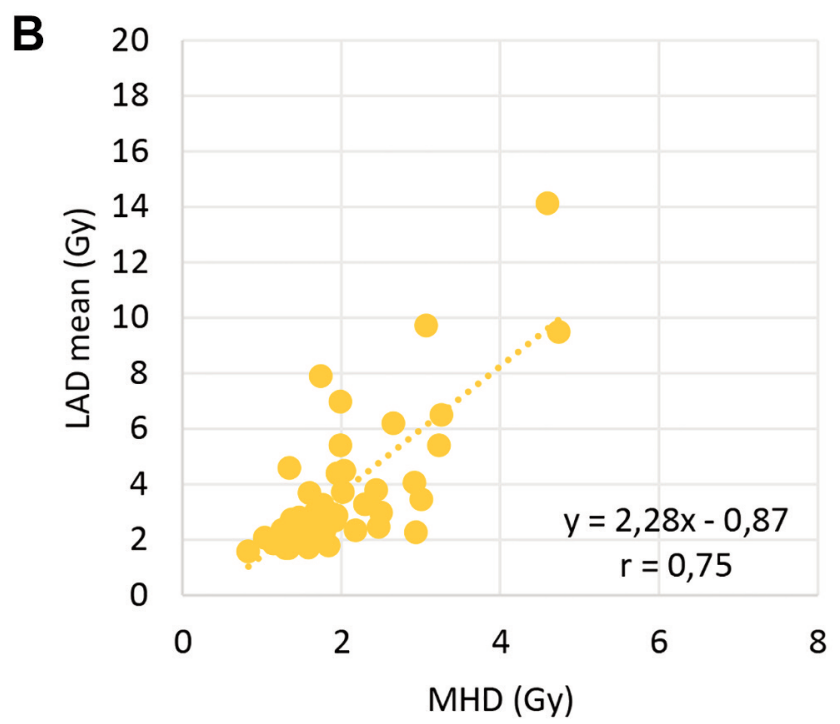

nDIBH HF $\quad \ldots \ldots \ldots$ Linear (nDIBH HF)

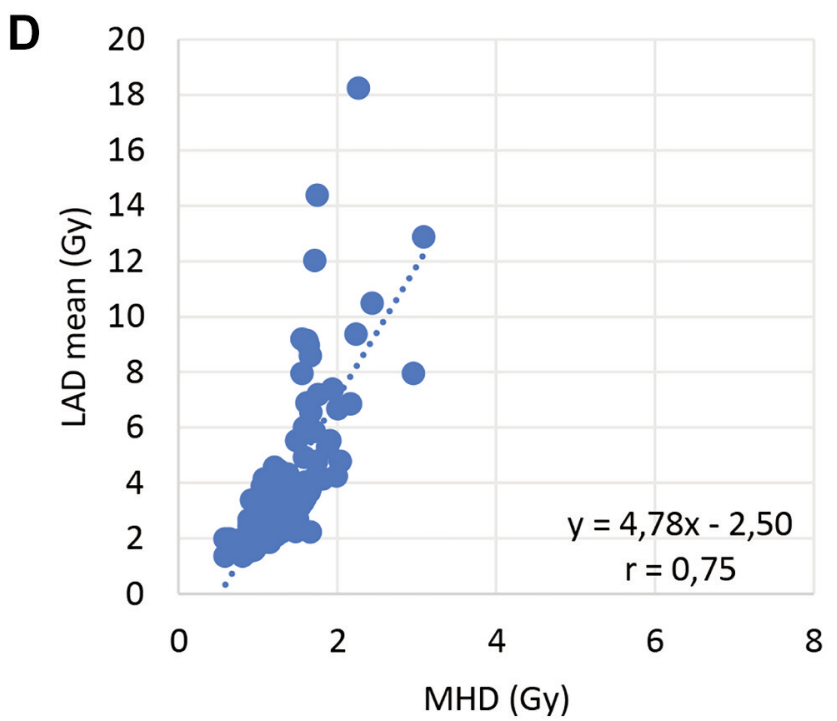

DIBH HF

Figure 2. Correlation between $L A D D_{\text {mean }}$ and MHD. (A) and (C) conventional fractionation without and with DIBH; $(B)$ and $(D)$ hypofractionation schedule without and with DIBH. LAD: Left anterior descending artery; MHD: mean heart dose; CF: conventional fractionation; HF: hypofractionated schedule; nDIBH: non-deep inspiration breath-hold; DIBH: deep inspiration breath-hold.

LAD $\mathrm{D}_{\text {mean }}$ was $3.75 \pm 2.45 \mathrm{~Gy}$ and in the HF/DIBH group $3.79 \pm 2.67$ Gy (Table IV).

In the $\mathrm{nDIBH}$ group the LAD $\mathrm{D}_{\max }$ was $12.43 \pm 12.18 \mathrm{~Gy}$ and in the DIBH group the LAD $\mathrm{D}_{\max }$ was $11.05 \pm 9.18 \mathrm{~Gy}$ (Table III). In the $\mathrm{CF} / \mathrm{nDIBH}$ group, LAD $\mathrm{D}_{\max }$ was $13.59 \pm 12.96 \mathrm{~Gy}$ and in the CF/DIBH group $13.43 \pm 10.31 \mathrm{~Gy}$.
In the $\mathrm{HF} / \mathrm{nDIBH}$ group, LAD $\mathrm{D}_{\max }$ was $8.82 \pm 8.47 \mathrm{~Gy}$ and in the HF/DIBH group 10.63 \pm 8.94 Gy (Table IV).

A positive linear correlation was found between LAD $\mathrm{D}_{\text {mean }}$ and MHD in all subgroups (Figure 2). In $\mathrm{nDIBH}$, the linear gradient between the LAD $\mathrm{D}_{\text {mean }}$ and MHD was 3.2 in $\mathrm{CF}$ and 2.3 in $\mathrm{HF}$, respectively. In $\mathrm{DIBH}$, the linear gradient 



- DIBH CF $\quad \ldots \ldots$ Linear (DIBH CF)

- DIBH HF $\quad \ldots \ldots$ L..... Linear (DIBH HF)

Figure 3. Correlation between $L A D D_{\max }$ and MHD. (A) and $(C)$ conventional fractionation without and with DIBH; $(B)$ and $(D)$ hypofractionation schedule without and with DIBH. Gy: Gray; LAD: left anterior descending artery; MHD: mean heart dose; CF: conventional fractionation; HF: hypofractionated schedule; nDIBH: non-deep inspiration breath-hold; DIBH: deep inspiration breath-hold.

between the LAD $D_{\text {mean }}$ and MHD was 2.5 in $\mathrm{CF}$ and 4.8 in HF. A high positive correlation (Pearson's correlation coefficient $r>0.7$ ) was found except in the subgroup $\mathrm{CF} / \mathrm{DIBH}$. Altogether, eight patients with a MHD below 3.0 Gy exhibited a LAD $\mathrm{D}_{\text {mean }}>10.0 \mathrm{~Gy}$ : four patients in subgroup $\mathrm{HF} / \mathrm{DIBH}$ and four patients in subgroup $\mathrm{CF} / \mathrm{nDIBH}$.
Our data showed a moderate linear correlation between LAD $D_{\max }$ and MHD in the $\mathrm{nDIBH}$ group as well as in the DIBH group (Figure 3). In the $\mathrm{nDIBH}$ group, the linear gradient between LAD $\mathrm{D}_{\max }$ and MHD was 6.2 in $\mathrm{CF}$ and 7.4 in $\mathrm{HF}$, respectively. In the $\mathrm{DIBH}$ group, the linear gradient between LAD $\mathrm{D}_{\max }$ and MHD was 12.9 in $\mathrm{CF}$ and 
15.8 in HF. In HF this resulted in a high positive correlation $(r>0.7$ in both $\mathrm{DIBH}$ and $\mathrm{nDIBH})$ whereas in $\mathrm{CF}$ this correlation was moderately only $(r=0.65$ in $\mathrm{DIBH}$ and $\mathrm{r}=0.63$ in $\mathrm{nDIBH})$.

Mean dose of the ipsilateral lung (MLD) was significantly higher in DIBH compared to $\mathrm{nDIBH}$ in both $\mathrm{CF}$ (DIBH $8.53 \pm 2.24$ Gy $v s$. nDIBH $7.03 \pm 2.94 \mathrm{~Gy} ; p=0.007$ ) as well as HF (DIBH 5.46 \pm 1.90 Gy vs. nDIBH 4.80 \pm 1.50 Gy; $p=0.017$ ) (Table IV). Similarly, ipsilateral lung volumes receiving at least 5 Gy, 10 Gy and 15 Gy (V5, V10, V15) were significantly higher in DIBH compared to $\mathrm{nDIBH}$ in both fractionation schemes $(p<0.05)$.

\section{Discussion}

Radiation exposure of the heart is a risk factor for the occurrence of coronary disease in oncological therapy. A recent analysis of a large data set from the Surveillance, Epidemiology, and End Results database (SEER) revealed an increased relative mortality in patients with left-sided breast cancer who received radiation before 1990 (7), especially in younger women and when chemotherapy was also given. After 1990 several studies showed no evidence of increased cardiac mortality for left-sided radiotherapy (8). However, there is still no proven relationship between the cardiac dose and the risk of cardiac mortality after several years of latency with an increase in relative risk of $4.1 \%$ per Gy mean cardiac dose (9). Pre-existing heart disease might increase the risk of acute cardiac events in a curative setting of oncologic treatment. In some cases, cardiac comorbidities may not be known at the time of treatment. Further systemic therapy (anthracyclines, taxanes, trastuzumab) increases the probability of cardiotoxicity (7). In these cases, it is essential to select patients, who could receive a significant dose to critical structures of the heart, namely the LAD, and then to offer them cardiac sparing radiotherapy with $e . g$., DIBH to avoid any additional increase in cardiac risk exposure.

Darby (3) found MHD being a predictor of major coronary events. This frequently cited study suggests that the risk of cardiac events is proportional to MHD with a relative increase of $7.4 \%$ per Gy of mean heart dose in the rate of major acute coronary events starting within the first five years after radiotherapy with no threshold. Considering this, our data showed that almost all patients (99\%) with DIBH had a mean MHD below 3.0 Gy and even 74\% patients with free breathing (nDIBH) had a mean MHD below 3.0 Gy. This already shows excellent heart protection in our cohort even before the publication of Darby (3). However, we found a significant further reduction of mean MHD from 2.64 Gray (Gy) in patients irradiated without DIBH compared to 1.39 Gy with DIBH (47\% relative reduction).

In a review by Drost et al. (10) the cardiac dose is described in case of whole breast irradiation except brachytherapy or proton radiotherapy between January 2014 and September 2017. On average, they could find that the MHD decreased between 2014 (4.6 Gy) and 2017 (2.6 Gy). Regimens with breathing control had a lower MHD (1.7 Gy) compared to regimens without breathing control (4.5 Gy). This is in line with our results as described above. A significant dose reduction due to breath-hold technique could be found in several studies with conventional fractionation regimens for MHD (11-13) as well as $\operatorname{LAD}(14,15)$.

When interpreting data from different treatment periods, it should be taken into account that nowadays almost exclusively hypofractionated treatment schedules are used. Our data showed a significant dose reduction in MHD in both conventional fractionation and hypofractionated schedules. Therefore, the existing dose recommendations for the heart need to be reconsidered in the era of hypofractionated schedules due to the radiobiological effect of fraction size. To correct dose distributions of hypofractionation treatment plans to equivalent dose in 2 Gy fractions, the linear quadratic model is used. This results in a lower biologically equivalent cardiac dose in HF compared to $\mathrm{CF}(16,17)$.

Furthermore, to date no increased cardiac mortality has been observed in the current studies on hypofractionation (18). Heart and LAD are located in the low dose range of the irradiation fields. While the heart is a complex organ at risk and $\alpha / \beta$ value should be used with caution (19), we need more long-term follow-up data in hypofractionated breast radiotherapy to adapt existing cardiac dose constraints especially in case of ultra-hypofractionated schedules.

This study found no significant reduction in $L A D D_{\text {mean }}$ by using DIBH. This might lead to the assumption, that DIBH technique may not necessarily lead to further reduction in the dose to the $\mathrm{LAD}$. However, we observed a correlation of LAD $\mathrm{D}_{\text {mean }}$ with MHD in all subgroups with a linear correlation coefficient $r>0.6$. Per $1.0 \mathrm{~Gy}$ in MHD the LAD $\mathrm{D}_{\text {mean }}$ increased by at least 2.0 Gy. This correlation was also described by Evans (20). Their data showed an increase in LAD $\mathrm{D}_{\text {mean }}$ by $4.82 \mathrm{~Gy}$ per $1.0 \mathrm{~Gy}$ in MHD. The authors concluded that the LAD may not need to be contoured separately when conventional tangential treatment technique is used. Having in mind that our collective of patients included some patients with high $L A D D_{\text {mean }}$ and LAD $D_{\max }$ despite low MHD, in our opinion it is not enough to contour the heart only as an organ at risk. Regarding the literature, it remains unclear, whether LAD $\mathrm{D}_{\text {mean }}$ is of relevance with respect to radiogenic cardiac events $(21,22)$. Based on this fact and on our findings, LAD should be additionally delineated as an organ at risk.

Furthermore, using MHD as the most important predictor for late cardiac toxicity disregards the fact that dose distribution within the heart is not homogenous. High radiation dose exposures can be observed in the apex of the 
heart as well as in the apical-interior segment, where the LAD originates $(23,24)$. In another study, an increase of stenosis in the LAD in hotspot areas resulting in an increased risk of coronary artery stenosis in the LAD was shown (25). This leads to the assumption that LAD $\mathrm{D}_{\max }$ may equally play an important role in the development of late cardiac events. Our data show a correlation between MHD and LAD $\mathrm{D}_{\max }$ in all subgroups. An increase of more than 6.0 Gy in LAD $D_{\max }$ per Gy MHD in all subgroups was seen. To avoid additional risk of adverse cardiac events, the hotspot areas to the LAD represented by $L A D D_{\max }$, should be kept as low as possible, accordingly.

Despite breath-hold technique, there is an intrinsic cardiac motion that should be considered in radiation planning. Wang (26) found that the major displacement of the heart occurring in the posterior area, which is unlikely to affect the heart dose received from tangential radiation. Displacement of the LAD was shown in $10 \%$ of the patients with at least $30 \%$ volume shift. This underlines the importance of protecting the LAD even when DIBH is used.

Due to the small volume of contoured LAD an underestimation of dose actually received to LAD may occur. There are several contouring atlases suggesting slightly different cardiac delineation. This results in a large variability in contouring heart and LAD. Lorenzen (27) showed large inter-observer variation in delineation of the LAD even using contouring guidelines. To enhance consistent delineation of organs at risk, in our study delineation of organs at risk (heart, LAD, lung) was performed by the same physician. This is in accordance to Feng (5) as well as other studies $(4,28-30)$. Nevertheless, our data show a large variance in volume of the LAD with an overall small volume of contoured LAD independent of the DIBH technique (nDIBH: $2.95 \pm 1.01 \mathrm{~cm}^{3}$, DIBH: $\left.2.9 \pm 0.71 \mathrm{~cm}^{3}\right)$. Additionally, the slice thickness of our planning CT-scans (3 mm) might have resulted in uncertainty in LAD delineation. Some authors (31) generated an additional planning structure around the LAD with a width of $5 \mathrm{~mm}$ anterior- posterior and $10 \mathrm{~mm}$ left-right around the LAD considering the uncertainties of contouring. They concluded that even rough contouring is sufficient to assess the dose of the LAD but with the recommendation to carefully contour the specific cardiac substructures such as LAD, while $\mathrm{D}_{\text {mean }}$ of the heart is not specific enough.

Dose distribution is also influenced by the radiation technique. Some studies show a dose reduction in heart and LAD using intensity modulated radiotherapy (IMRT) plans $(32,33)$. In elderly breast cancer patients, there was shown a significant dose reduction in $L A D D_{\text {mean }}$ and LAD $D_{\max }$ without a significant difference in heart sparing (34). Despite a low MHD achievable in IMRT plans there is still a risk of hot spots within the LAD making additional contouring of LAD mandatory for IMRT plan optimization (35). Mast (29) demonstrated that the reduction in LAD $\mathrm{D}_{\text {mean }}$ using DIBH was marginally greater in 3D-CRT compared with IMRT.

Overall, there is a small difference between 3D-CRT and IMRT in terms of reducing MHD and LAD $\mathrm{D}_{\text {mean }}$ using DIBH. In the era of IMRT and volumetric arc therapy (VMAT) techniques, it is justified to use conventional tangential treatment technique because the modern techniques take longer in applying irradiation and the patients need to hold their breath for a longer time. In some selected cases it might be well founded to use IMRT or VMAT technique to lower the dose to the heart as well to the LAD. Using IMRT or VMAT, it should be kept in mind that there can potentially be an increased risk of secondary cancers due to change of dose distribution and increase in monitor units (36). According to national treatment guidelines (37), radiation with tangential fields is still considered standard of care and IMRT techniques should be limited to patients with special conditions like larger breast volume or deviating anatomy of breast or thoracic curvature.

Apart from the heart, the lungs are also irradiated during tangential chest irradiation possibly leading to development of pulmonary fibrosis and lung function decline. In our analysis, the lung volume was significantly larger in patients with DIBH compared to those without DIBH $(2,102 \pm 424$ $\mathrm{cm}^{3}$ respectively $1,223 \pm 323 \mathrm{~cm}^{3}$ ) due to the anatomical position changes during deep inspiration. The mean lung dose (MLD) was also significantly higher in DIBH in both HF (5.46 $\pm 1.90 \mathrm{~Gy}$ in DIBH and 4.8 \pm 1.50 in $\mathrm{nDIBH})$ and $\mathrm{CF}$ $(8.53 \pm 2.24$ Gy in DIBH and 7.03 $\pm 2.94 \mathrm{~Gy}$ in $\mathrm{nDIBH})$. In the HF group, however, the MLD was lower overall compared to the CF group.

Chan (11) summarizes in a review article several studies concerning this topic. The literature contains contradicting results regarding the resulting lung doses when using DIBH. Quantitative dose-volume analyses do not take into account different density of the lung between inspiration and expiration. With increasing lung volume in deep inspiration, the lung density may decrease, resulting in irradiation of a reduced fraction of normal lung mass (37). To avoid mistakes in interpretation of dose-volume constraints to the lung, Oechsner (41) suggests the dose-mass histogram $(\mathrm{DMH})$ as a more accurate model to measure relevant dose to the lung. In that study, the mean lung density was decreased by using DIBH technique compared to free breathing. This is in contrast to the results of our analysis, which showed an increased MLD of the lung using DIBH in both HF and CF. Possibly, there might be differences between techniques of breathing maneuvers. In 2018, Zhao (42) presented a prospective analysis showing the abdominal breathing maneuver as the most lung and cardiac sparing technique compared to thoracic breathing. To generate comparable data, there should be consistent requirements of the breathing maneuver. Finally, it is not clearly understood 
in the current literature whether the lung dose and therefore the risk of radiation-induced pneumonitis is increased using DIBH. Furthermore, as the purpose for DIBH treatment is to avoid heart dose, it is also important to point out that DIBH may result in increased pulmonary exposure, which may lead to corresponding pulmonary toxicity.

Our results regarding dose distribution of the heart are in line with Ferini (43) showing a significant dose reduction of MHD as well as LAD $\mathrm{D}_{\text {mean }}$ in DIBH technique compared to $\mathrm{nDIBH}$. The distance between the tangent fields in case of 3D-CRT is the most important influencing factor for dose distribution to the heart. It can be used as an indicator for the actual benefit in terms of heart protection. The authors conclude that with the help of this anatomical predictor, the decision to perform irradiation in DIBH can be facilitated and objectified.

The presented study was performed retrospectively. Known limitations of retrospective studies include the quality of available data, which in our case were collected up to 10 years ago. To eliminate the differences regarding the contouring risk organs heart and lung, all planning data were re-contoured by the same physician. This results in comparable data from the two periods analyzed.

Another disadvantage of retrospective studies is bias due to confounding variables. Patients of this study were clustered in two groups that represent different periods. In the first period (2011-2013), irradiation in CF was state of the art and mainly used. Subsequent evolvement of irradiation technique led to changes in the second period (2017-2019) where more often HF was used. Considering this confounding variable our analyses was done in subgroups comparing $\mathrm{DIBH} / \mathrm{HF}$ with $\mathrm{nDIBH} / \mathrm{HF}$ and $\mathrm{DIBH} / \mathrm{CF}$ with $\mathrm{nDIBH} / \mathrm{CF}$.

Besides the retrospective nature, the limitation of this study is the inter-individual comparison of dose-volume parameters irradiated in two different treatment periods. In contrast, other authors did intra-individual dosimetric comparison of treatment plans but equally found a significant decrease of cardiac dose $(13,37-40)$. Compared to other publications regarding DIBH in our study is included a large number of patients, which we see as an advantage.

In conclusion, DIBH could significantly reduce the MHD in conventional fractionated and hypofractionated scheduled radiotherapy. DIBH is a simple and reproducible technique and is readily applicable in routine clinical practice. However, it does not seem sufficient to focus attention merely on MHD, because hotspots may occur at LAD even though MHD is low. Consistent data with comparable groups of patients and breathing maneuvers should be sampled to generate standardized dose constraints, taking into account the individual patient characteristics such as age, compliance and co-morbidities as well as fractionation schedule and modern irradiation techniques such as IMRT. There is a need to define valid criteria to select patients who benefit most from DIBH in terms of mean heart dose.

\section{Conflicts of Interest}

The Authors declare no conflicts of interest in association with the present study.

\section{Authors' Contributions}

Anne Caroline Knöchelmann collected the data, analysed them statistically, put the results into the current context and wrote the article. Nese Ceylan researched part of the patients who were eligible for the study. Michael Bremer revised it critically for important intellectual content. All Authors read and approved the final version.

\section{References}

1 Siegel RL, Miller KD, Fuchs HE and Jemal A: Cancer statistics, 2021. CA Cancer J Clin 71(1): 7-33, 2021. PMID: 33433946. DOI: $10.3322 /$ caac. 21654

2 Clarke M, Collins R, Darby S, Davies C, Elphinstone P, Evans V, Godwin J, Gray R, Hicks C, James S, MacKinnon E, McGale P, McHugh T, Peto R, Taylor C, Wang Y and Early Breast Cancer Trialists' Collaborative Group (EBCTCG): Effects of radiotherapy and of differences in the extent of surgery for early breast cancer on local recurrence and 15-year survival: an overview of the randomised trials. Lancet 366(9503): 2087-2106, 2005. PMID: 16360786. DOI: 10.1016/S0140-6736(05)67887-7

3 Darby SC, Ewertz M, McGale P, Bennet AM, Blom-Goldman U, Brønnum D, Correa C, Cutter D, Gagliardi G, Gigante B, Jensen MB, Nisbet A, Peto R, Rahimi K, Taylor C and Hall P: Risk of ischemic heart disease in women after radiotherapy for breast cancer. N Engl J Med 368(11): 987-998, 2013. PMID: 23484825. DOI: 10.1056/NEJMoa1209825

4 Yeung R, Conroy L, Long K, Walrath D, Li H, Smith W, Hudson A and Phan T: Cardiac dose reduction with deep inspiration breath hold for left-sided breast cancer radiotherapy patients with and without regional nodal irradiation. Radiat Oncol 10: 200, 2015. PMID: 26391237. DOI: 10.1186/s13014015-0511-8

5 Feng M, Moran JM, Koelling T, Chughtai A, Chan JL, Freedman L, Hayman JA, Jagsi R, Jolly S, Larouere J, Soriano J, Marsh R and Pierce LJ: Development and validation of a heart atlas to study cardiac exposure to radiation following treatment for breast cancer. Int J Radiat Oncol Biol Phys 79(1): 10-18, 2011. PMID: 20421148. DOI: 10.1016/j.ijrobp.2009.10.058

6 White J, Tai A, Arthur D, Buchholz T, MacDonald S, Marks L, Pierce L, Recht A, Rabinovitch R, Taghian A, Vicini F, Woodward W and Li XA: Breast Cancer Atlas for Radiation Therapy Planning: Consensus Definitions. Available at: https://www.nrgoncology.org/Portals/0/Scientific\%20Program/CI RO/Atlases/BreastCancerAtlas_corr.pdf?ver=2018-04-18144201-270 [Last accessed on June 15, 2021]

7 Henson KE, McGale P, Darby SC, Parkin M, Wang Y and Taylor CW: Cardiac mortality after radiotherapy, chemotherapy and endocrine therapy for breast cancer: Cohort study of 2 million 
women from 57 cancer registries in 22 countries. Int J Cancer 147(5): 1437-1449, 2020. PMID: 32022260. DOI: 10.1002/ ijc. 32908

8 Weberpals J, Jansen L, Müller OJ and Brenner H: Long-term heart-specific mortality among 347476 breast cancer patients treated with radiotherapy or chemotherapy: a registry-based cohort study. Eur Heart J 39(43): 3896-3903, 2018. PMID: 29635274. DOI: 10.1093/eurheartj/ehy167

9 Taylor C, Correa C, Duane FK, Aznar MC, Anderson SJ, Bergh J, Dodwell D, Ewertz M, Gray R, Jagsi R, Pierce L, Pritchard KI, Swain S, Wang Z, Wang Y, Whelan T, Peto R, McGale P and Early Breast Cancer Trialists' Collaborative Group: Estimating the risks of breast cancer radiotherapy: Evidence from modern radiation doses to the lungs and heart and from previous randomized trials. J Clin Oncol 35(15): 1641-1649, 2017. PMID: 28319436. DOI: $10.1200 / J C O .2016 .72 .0722$

10 Drost L, Yee C, Lam H, Zhang L, Wronski M, McCann C, Lee J, Vesprini D, Leung E and Chow E: A systematic review of heart dose in breast radiotherapy. Clin Breast Cancer 18(5): e819-e824, 2018. PMID: 29980429. DOI: 10.1016/j.clbc. 2018.05.010

11 Chan TY, Tang JI, Tan PW and Roberts N: Dosimetric evaluation and systematic review of radiation therapy techniques for early stage node-negative breast cancer treatment. Cancer Manag Res 10: 4853-4870, 2018. PMID: 30425577. DOI: 10.2147/CMAR.S172818

12 Nissen HD and Appelt AL: Improved heart, lung and target dose with deep inspiration breath hold in a large clinical series of breast cancer patients. Radiother Oncol 106(1): 28-32, 2013. PMID: 23199652. DOI: 10.1016/j.radonc.2012.10.016

13 Lastrucci L, Borghesi S, Bertocci S, Gasperi C, Rampini A, Buonfrate G, Pernici P, De Majo R and Gennari PG: Advantage of deep inspiration breath hold in left-sided breast cancer patients treated with 3D conformal radiotherapy. Tumori 103(1): 72-75, 2017. PMID: 27716875. DOI: 10.5301/tj.5000563

14 Poitevin-Chacón MA, Ramos-Prudencio R, Rumoroso-García JA, Rodríguez-Laguna A and Martínez-Robledo JC: Voluntary breath-hold reduces dose to organs at risk in radiotherapy of leftsided breast cancer. Rep Pract Oncol Radiother 25(1): 104-108, 2020. PMID: 31908602. DOI: 10.1016/j.rpor.2019.12.016

15 Becker-Schiebe M, Stockhammer M, Hoffmann W, Wetzel F and Franz H: Does mean heart dose sufficiently reflect coronary artery exposure in left-sided breast cancer radiotherapy?: Influence of respiratory gating. Strahlenther Onkol 192(9): 624631, 2016. PMID: 27389036. DOI: 10.1007/s00066-016-1011-y

16 Loap P and Kirova Y: The challenge of cardiac dose constraint adaptation to hypofractionated breast radiotherapy in clinical practice. Strahlenther Onkol 197(6): 555-557, 2021. PMID: 33891125. DOI: $10.1007 / \mathrm{s} 00066-021-01777-2$

17 Appelt AL, Vogelius IR and Bentzen SM: Modern hypofractionation schedules for tangential whole breast irradiation decrease the fraction size-corrected dose to the heart. Clin Oncol (R Coll Radiol) 25(3): 147-152, 2013. PMID: 22910644. DOI: 10.1016/j.clon.2012.07.012

18 Haviland JS, Owen JR, Dewar JA, Agrawal RK, Barrett J, Barrett-Lee PJ, Dobbs HJ, Hopwood P, Lawton PA, Magee BJ, Mills J, Simmons S, Sydenham MA, Venables K, Bliss JM, Yarnold JR and START Trialists' Group: The UK Standardisation of Breast Radiotherapy (START) trials of radiotherapy hypofractionation for treatment of early breast cancer: 10-year follow-up results of two randomised controlled trials. Lancet Oncol 14(11): 1086-1094, 2013. PMID: 24055415. DOI: $10.1016 / \mathrm{S} 1470-2045(13) 70386-3$

19 Loap P, Fourquet A and Kirova Y: The limits of the linear quadratic (LQ) model for late cardiotoxicity prediction: Example of hypofractionated rotational intensity modulated radiation therapy (IMRT) for breast cancer. Int J Radiat Oncol Biol Phys 106(5): 1106-1108, 2020. PMID: 31973883. DOI: 10.1016/ j.ijrobp.2019.12.006

20 Evans SB, Panigrahi B, Northrup V, Patterson J, Baldwin DE, Higgins SA and Moran MS: Analysis of coronary artery dosimetry in the 3-dimensional era: Implications for organ-atrisk segmentation and dose tolerances in left-sided tangential breast radiation. Pract Radiat Oncol 3(2): e55-e60, 2013. PMID: 24674321. DOI: $10.1016 /$ j.prro.2012.06.007

21 Borger JH, Hooning MJ, Boersma LJ, Snijders-Keilholz A, Aleman BM, Lintzen E, van Brussel S, van der Toorn PP, Alwhouhayb $M$ and van Leeuwen FE: Cardiotoxic effects of tangential breast irradiation in early breast cancer patients: the role of irradiated heart volume. Int J Radiat Oncol Biol Phys 69(4): 1131-1138, 2007. PMID: 17606332. DOI: 10.1016/ j.ijrobp.2007.04.042

22 Gagliardi G, Constine LS, Moiseenko V, Correa C, Pierce LJ, Allen AM and Marks LB: Radiation dose-volume effects in the heart. Int J Radiat Oncol Biol Phys 76(3 Suppl): S77-S85, 2010. PMID: 20171522. DOI: 10.1016/j.ijrobp.2009.04.093

23 Moignier A, Broggio D, Derreumaux S, El Baf F, Mandin AM, Girinsky T, Paul JF, Chea M, Jenny C, Franck D, Aubert B and Mazeron JJ: Dependence of coronary 3-dimensional dose maps on coronary topologies and beam set in breast radiation therapy: a study based on CT angiographies. Int J Radiat Oncol Biol Phys 89(1): 182-190, 2014. PMID: 24725701. DOI: 10.1016/j.ijrobp. 2014.01.055

24 Correa CR, Litt HI, Hwang WT, Ferrari VA, Solin LJ and Harris EE: Coronary artery findings after left-sided compared with right-sided radiation treatment for early-stage breast cancer. J Clin Oncol 25(21): 3031-3037, 2007. PMID: 17634481. DOI: 10.1200/JCO.2006.08.6595

25 Nilsson G, Holmberg L, Garmo H, Duvernoy O, Sjögren I, Lagerqvist B and Blomqvist C: Distribution of coronary artery stenosis after radiation for breast cancer. J Clin Oncol 30(4): 380386, 2012. PMID: 22203772. DOI: 10.1200/JCO.2011.34.5900

26 Wang X, Pan T, Pinnix C, Zhang SX, Salehpour M, Sun TL, Gladish G, Strom EA, Perkins GH, Tereffe W, Woodward W, Hoffman KE, Buchholz TA and Yu TK: Cardiac motion during deep-inspiration breath-hold: implications for breast cancer radiotherapy. Int J Radiat Oncol Biol Phys 82(2): 708-714, 2012. PMID: 21444161. DOI: 10.1016/j.ijrobp.2011.01.035

27 Lorenzen EL, Taylor CW, Maraldo M, Nielsen MH, Offersen BV, Andersen MR, O'Dwyer D, Larsen L, Duxbury S, Jhitta B, Darby SC, Ewertz $M$ and Brink C: Inter-observer variation in delineation of the heart and left anterior descending coronary artery in radiotherapy for breast cancer: a multi-centre study from Denmark and the UK. Radiother Oncol 108(2): 254-258, 2013. PMID: 23891098. DOI: 10.1016/j.radonc.2013.06.025

28 Lee HY, Chang JS, Lee IJ, Park K, Kim YB, Suh CO, Kim JW and Keum KC: The deep inspiration breath hold technique using Abches reduces cardiac dose in patients undergoing left-sided breast irradiation. Radiat Oncol J 31(4): 239-246, 2013. PMID: 24501713. DOI: $10.3857 /$ roj.2013.31.4.239 
29 Mast ME, van Kempen-Harteveld L, Heijenbrok MW, Kalidien Y, Rozema H, Jansen WP, Petoukhova AL and Struikmans H: Left-sided breast cancer radiotherapy with and without breathhold: does IMRT reduce the cardiac dose even further? Radiother Oncol 108(2): 248-253, 2013. PMID: 24044804. DOI: 10.1016/j.radonc.2013.07.017

30 Lawler $G$ and Leech M: Dose sparing potential of deep inspiration breath-hold technique for left breast cancer radiotherapy organs-at-risk. Anticancer Res 37(2): 883-890, 2017. PMID: 28179347. DOI: 10.21873/anticanres.11394

31 Duma MN, Herr AC, Borm KJ, Trott KR, Molls M, Oechsner $\mathrm{M}$ and Combs SE: Tangential field radiotherapy for breast cancer-the dose to the heart and heart subvolumes: What structures must be contoured in future clinical trials? Front Oncol 7: 130, 2017. PMID: 28674678. DOI: 10.3389/fonc. 2017.00130

32 Sripathi LK, Ahlawat P, Simson DK, Khadanga CR, Kamarsu L, Surana SK, Arasu K and Singh H: Cardiac dose reduction with deep-inspiratory breath hold technique of radiotherapy for leftsided breast cancer. J Med Phys 42(3): 123-127, 2017. PMID: 28974856. DOI: 10.4103/jmp.JMP_139_16

33 Nieder C, Schill S, Kneschaurek P and Molls M: Influence of different treatment techniques on radiation dose to the LAD coronary artery. Radiat Oncol 2: 20, 2007. PMID: 17547777. DOI: $10.1186 / 1748-717 \mathrm{X}-2-20$

34 Carosi A, Ingrosso G, Turturici I, Valeri S, Barbarino R, Di Murro L, Bottero M, Lancia A, Ponti E, Bruni A, Bonzano E, Saldi S, Andolina M, Aristei C and Santoni R: Whole breast external beam radiotherapy in elderly patients affected by leftsided early breast cancer: a dosimetric comparison between two simple free-breathing techniques. Aging Clin Exp Res 32(7): 1335-1341, 2020. PMID: 31429001. DOI: 10.1007/s40520-01901312-5

35 Testolin A, Ciccarelli S, Vidano G, Avitabile R, Dusi F and Alongi F: Deep inspiration breath-hold intensity modulated radiation therapy in a large clinical series of 239 left-sided breast cancer patients: a dosimetric analysis of organs at risk doses and clinical feasibility from a single center experience. Br J Radiol 92(1101): 20190150, 2019. PMID: 31265316. DOI: 10.1259/ bjr.20190150

36 Haciislamoglu E, Colak F, Canyilmaz E, Zengin AY, Yilmaz AH, Yoney A and Bahat Z: The choice of multi-beam IMRT for whole breast radiotherapy in early-stage right breast cancer. Springerplus 5(1): 688, 2016. PMID: 27350922. DOI: $10.1186 / \mathrm{s} 40064-016-2314-2$
37 AWMF 2020: Langfassung der Leitlinie "Früherkennung, Diagnostik, Therapie und Nachsorge des Mammakarzinoms". Available at: https://www.awmf.org/uploads/tx_szleitlinien/032045OLl_S3_Mammakarzinom_2020-02.pdf [Last accessed on June 15, 2021]

38 Sixel KE, Aznar MC and Ung YC: Deep inspiration breath hold to reduce irradiated heart volume in breast cancer patients. Int $\mathrm{J}$ Radiat Oncol Biol Phys 49(1): 199-204, 2001. PMID: 11163515. DOI: 10.1016/s0360-3016(00)01455-3

39 Lu HM, Cash E, Chen MH, Chin L, Manning WJ, Harris J and Bornstein B: Reduction of cardiac volume in left-breast treatment fields by respiratory maneuvers: a CT study. Int J Radiat Oncol Biol Phys 47(4): 895-904, 2000. PMID: 10863057. DOI: $10.1016 / \mathrm{s} 0360-3016(00) 00512-5$

40 Joo JH, Kim SS, Ahn SD, Kwak J, Jeong C, Ahn SH, Son BH and Lee JW: Cardiac dose reduction during tangential breast irradiation using deep inspiration breath hold: a dose comparison study based on deformable image registration. Radiat Oncol 10: 264, 2015. PMID: 26715382. DOI: 10.1186/s13014-015-0573-7

41 Oechsner M, Düsberg M, Borm KJ, Combs SE, Wilkens JJ and Duma MN: Deep inspiration breath-hold for left-sided breast irradiation: Analysis of dose-mass histograms and the impact of lung expansion. Radiat Oncol 14(1): 109, 2019. PMID: 31215458. DOI: 10.1186/s13014-019-1293-1

42 Zhao F, Shen J, Lu Z, Luo Y, Yao G, Bu L, Ge J, Yang X, Ning $\mathrm{L}$ and Yan S: Abdominal DIBH reduces the cardiac dose even further: a prospective analysis. Radiat Oncol 13(1): 116, 2018. PMID: 29929560. DOI: 10.1186/s13014-018-1062-6

43 Ferini G, Molino L, Tripoli A, Valenti V, Illari SI, Marchese VA, Cravagno IR and Borzi GR: Anatomical predictors of dosimetric advantages for deep-inspiration-breath-hold 3D-conformal radiotherapy among women with left breast cancer. Anticancer Res 41(3): 1529-1538, 2021. PMID: 33788746. DOI: 10.21873/ anticanres.14912
Received August 27, 2021 Revised September 10, 2021 Accepted October 11, 2021 